\title{
Towards more biological mutation operators in gene regulation studies
}

\author{
James Watson $^{\mathrm{a}}$, Nicholas Geard ${ }^{\mathrm{a}}$ and Janet Wiles ${ }^{\mathrm{a}, \mathrm{b}}$

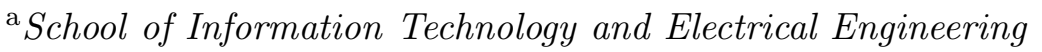 \\ ${ }^{\mathrm{b}}$ School of Psychology \\ The University of Queensland, Australia
}

\begin{abstract}
Genetic regulation is often viewed as a complex system whose properties emerge from the interaction of regulatory genes. One major paradigm for studying the complex dynamics of gene regulation uses directed graphs to explore structure, behaviour and evolvability. Mutation operators used in such studies typically involve the insertion and deletion of nodes, and the insertion, deletion and rewiring of links at the network level. These network-level mutational operators are sufficient to allow the statistical analysis of network structure, but impose limitations on the way networks are evolved. There are a wide variety of mutations in DNA sequences that have yet to be analysed for their network-level effects.

By modelling an artificial genome at the level of nucleotide sequences and mapping it to a regulatory network, biologically grounded mutation operators can be mapped to network level mutations. This paper analyses five such sequence level mutations (single-point mutation, transposition, inversion, deletion and gene duplication) for their effects at the network level. Using analytic and simulation techniques, we show that it is rarely the case that nodes and links are cleanly added or deleted, with even the simplest point mutation causing a wide variety of network-level modifications. As expected, the vast majority of simple (single-point) mutations are neutral, resulting in a neutral plateau from which a range of functional behaviours can be reached.

By analysing the effects of sequence-level mutations at the network level of gene regulation, we aim to stimulate more careful consideration of mutation operators in gene regulation models than has previously been given.
\end{abstract}

Key words: gene regulation, Random Boolean Networks, mutation operators, simulation, artificial genome, evolutionary algorithms, molecular evolution and theoretical biology

Email addresses: jwatson@itee.uq.edu.au (James Watson), nic@itee.uq.edu.au (Nicholas Geard), j.wiles@itee.uq.edu.au (Janet Wiles). 


\section{Evolutionary modelling of genetic regulatory networks}

Evolution is a process of change and selection. Most spontaneous changes to the genome are corrected by DNA repair mechanisms, however these occasionally fail and result in a permanent mutation. A variety of different types of mutation are possible, ranging from point mutations of individual bases to rearrangements such as deletion and duplication and the movement of transposons. One view of the evolution of the genome holds that point mutations enable 'fine tuning', but that more radical changes, such as gene duplication, are necessary for its long term development (Alberts et al., 1994).

It has become increasingly common to view the genome as a complex system, whose properties emerge from the behaviour of networks of interacting genes (Kauffman, 1993). While a large part of molecular biology is dedicated to understanding the genome at the level of individual genes and the mechanisms by which they operate, there is a growing awareness that there are many emergent properties of the genome that are not reducible to the properties of its constituent elements (Solé and Goodwin, 2000). As the network view of the genome becomes more accepted, new abstract models have been developed to investigate its structure, dynamics and evolution.

Almost all of these models use a network level description of the genome. While this approach is sufficient to allow statistical analysis of network structure (Jeong et al., 2000; Wagner and Fell, 2001) and investigation of global patterns of behaviour (Kauffman, 1971, 1993), it imposes limitations on the way networks are evolved. Changes that can be made to networks are constrained to the addition and deletion of nodes, and the addition, deletion and rewiring of links.

When using artificial evolution to model a biological system, the choice of mutation operators should reflect the constraints of the problem being modelled. Biological mutations act upon the underlying nucleotide sequence; changes at this level do not necessarily have a one-to-one correspondence with network changes. Therefore, the mutation operators currently used to evolve artificial genetic regulatory networks (GRNs) represent only a subset of the types of mutations that occur in nature.

Recently, a new approach to modelling GRNs was developed in which a genome is represented as a sequence, analogous to the string of nucleotides forming a real genome. A GRN can then be extracted from this sequence using a simple set of rules based on biological mechanisms (Reil, 1999). An important, but currently unexplored, advantage of this approach is that it allows a range of mutation operators to be applied to the genome sequence whose effect can then be observed at the level of the regulatory network. The aim of this study was 
to explore and analyse the effect that various types of sequence-level mutation operators have on the resulting genetic regulatory networks.

Sections 2 reviews current approaches to evolving GRNs via mutation and selection. Section 3 outlines the types of mutation possible in biological systems. Section 4 then describes Reil's Artificial Genome model. Section 5 is an analysis of the network-level effects of five types of sequence-level change: singlepoint mutation, duplication, inversion, deletion and transposition. Section 6 describes the cumulative effects of applying duplications and single-point mutations over evolutionary time. Finally, the implications of these results for GRN modelling are discussed.

\section{Current approaches to evolving gene regulatory networks}

A number of different approaches have been taken to the abstract modelling of GRNs (Bornholdt, 2001). This section reviews several typical approaches in which the models have been subjected to some form of artificial evolution and focuses on the types of mutation operators that have been used to generate genotypic novelty.

One of the first attempts to treat GRNs as an instance of a complex system was made by Kauffman, using the Random Boolean Network abstraction (Kauffman, 1971). In this framework, genes were viewed as Boolean switches whose state was either on or off depending on whether or not they were being transcribed. Each gene in a system took as its input the states of a subset of other genes in the network and used a Boolean function to determine its output on the basis of these inputs (see Figure 1). The structural features of interest in these systems were the number of genes $(N)$ and the average number of inputs to a gene $(K)$. The dynamic feature of interest was the global pattern of behaviour, which could be quantified by such properties as the time taken to reach a stable pattern of expression, the length of cyclic expression patterns and the number of such patterns that could coexist in a given system.

For the purposes of artificial evolution, these networks are frequently represented by two data structures:

(1) A connection matrix specifying the structure of the network, where a ' +1 ' in the cell $(i, j)$ specifies a positive regulatory interaction between node $i$ and $\mathrm{j}$, ' -1 ' specifies a negative interaction, and ' 0 ' indicates no connection exists.

(2) A set of Boolean functions, one for each node, determining the dynamics of the network. 


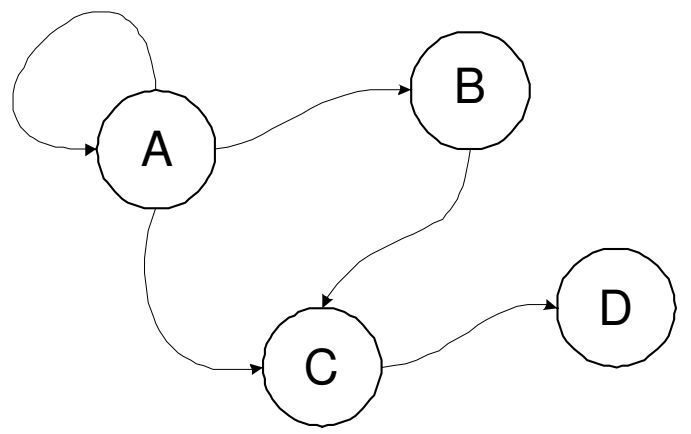

Boolean activation

function for node $\mathrm{C}$

\begin{tabular}{ll|l}
$\mathrm{A}$ & $\mathrm{B}$ & $\mathrm{C}$ \\
\hline 0 & 0 & 0 \\
0 & 1 & 1 \\
1 & 0 & 1 \\
1 & 1 & 0
\end{tabular}

Fig. 1. Random Boolean Networks. An RBN represents genes as Boolean nodes, connected into a network structure by regulatory interactions. In the network above, node $\mathrm{C}$ is regulated by nodes $\mathrm{A}$ and $\mathrm{B}$, and it in turn regulates node $\mathrm{D}$. A node is updated according to a Boolean function. An example function is shown on the right. The bottom line encodes the rule that, if nodes $\mathrm{A}$ and $\mathrm{B}$ are both on (i.e., being expressed), node $\mathrm{C}$ will be off (i.e., not expressed) in the next time step.

It is obvious that there are two types of mutation that can be made to such a system: (a) structural mutations, resulting in the addition or deletion of a node or link; and (b) mutations to the Boolean function determining the output of a node, resulting in a change in the dynamics of the network. These forms of mutation have been used in a wide variety of different gene network studies investigating evolvability (Kauffman and Smith, 1986; Kauffman, 1990; Frank, 1999), robustness to perturbation (Bornholdt and Sneppen, 2000), robustness to noise (Stern, 1999), evolutionary dynamics (Bornholdt and Sneppen, 1998; Bornholdt and Rohlf, 2000) and the structure of attractors (Wuensche, 1998).

A common methodology used to evolve these networks is to apply a genetic algorithm (GA) (Mitchell, 1996). Using this method, the connection matrix and Boolean functions are unfolded into a binary string, to which standard GA operators (such as point mutation and crossover) can be applied. A major problem with this approach is its lack of biological realism. In biology, network connectivity and function are not necessarily found in specific loci in the sequence, but rather derived from multiple sources spread across the genome. Hence, although inspired by biological events, the mutation operators applied to this representation have limited biological significance in this context.

A second paradigm for investigating genetic systems utilises tools and techniques developed to investigate the class of complex networks initially termed small-world networks (Watts, 1999; Strogatz, 2001). A recent surge of interest in complex networks led to the realisation that food webs, genetic and metabolic networks, neural networks, electrical power grids and the World Wide Web all share similar topological features (Strogatz, 2001). Rather than being completely ordered lattices, or chaotic random graphs, these networks seem to fall into a category somewhere in between, characterised by a short average path length (the number of links between any two nodes) and a high 
clustering coefficient (i.e., if $\mathrm{A}$ is linked to $\mathrm{B}$ and $\mathrm{B}$ is linked to $\mathrm{C}$, it is likely that $\mathrm{A}$ is also linked to $\mathrm{C}$ ).

A further characteristic possessed by many of these networks is that the number of links connecting each node follows a power law. These were termed scale-free networks due to the fact that their statistical properties remain constant as their size increases, and they possess several additional properties such as being tolerant to error (random removal of nodes) but vulnerable to attack by targeted removal of highly connected nodes (Albert et al., 2000). Analyses of experimental data gathered on the metabolic networks of a number of organisms revealed a high degree of correspondence between these theoretical models and the real world (Jeong et al., 2000; Wagner and Fell, 2001; Wolf et al., 2002). In particular it was found that the way in which the networks in these abstract models "grow" by the addition of new nodes and links could cast light on how biological networks may have evolved.

One of the most straightforward methods of growing artificial networks with small-world and scale-free properties is to add new nodes and preferentially link them to existing nodes that already have a large number of links (Dorogovtsev and Mendes, 2002). Genetic networks, by comparison, are thought to have grown to their current size largely due to the repeated duplication of genes and their subsequent diversification into new functional domains (Ohno, 1970; Lynch, 2002). Several models of how genetic networks may evolve via gene duplication have been developed and found to produce networks matching at least some of the properties of biological networks (Wagner, 1994; Vázquez et al., 2001; Solé et al., 2002; Pastor-Satorras et al., 2003). In these models, gene duplication is generally implemented by copying a node in the network along with all its links. Diversification is modelled by probabilistically rewiring these links, in some cases deriving the relevant parameter values from biological data (Solé et al., 2002).

While this method of modelling captures one aspect of the biological process in question, the exploration of new functional space via the diversification of duplicate genes, it fails to capture other aspects, such as potential damage to the target region of the genome, and the frequency with which particular network changes are likely to occur. The following two sections briefly review how mutation occurs in biology and describe how this process could more meaningfully be implemented in an abstract model.

\section{Mutation in biological systems}

Mutations are spontaneous changes to chromosomes that are not corrected by DNA repair mechanisms. These changes can arise in the DNA replication 
process, as a result of recombination events and also as a result of the actions of transposons. The rate at which mutations occur is generally very low (approximately $10^{-4}$ to $10^{-6}$ mutations per gene per generation in humans) but can be increased by environmental influences, such as chemicals, radiation and transposable elements.

The simplest class of mutation is a point mutation, in which a single nucleotide in a sequence is altered. This type of mutation generally has a localised effect on the activity of a single gene. A second class of mutations involves more radical restructuring of a chromosome:

- Duplication: an increase in the number of copies of a segment of a chromosome. Duplication events can be either tandem, in which case the copied region adjoins the original region, or non-tandem, where the copied region may be in another part of the chromosome or on another chromosome altogether.

- Deletion: the removal of a segment of a chromosome. Deletion events can be intragenic, affecting only one gene, or multigenic, affecting a number of genes.

- Transposition: removing a segment from the end of one chromosome and re-attaching it to another.

- Inversion: the reversal of a segment of a chromosome.

A final class of mutations results from the actions of transposable elements. Transposons exist in large numbers in eukaryotic genomes and are capable of leaving one site, multiplying and spreading to many other sites. Mutations can result both from the insertion of a transposon into a gene and from modifications made to the gene sequence when it leaves. Even if a transposon does not affect the coding region of a gene (and hence the structure of its RNA or protein product), it may affect the activation of a gene by altering its regulatory region (which may contain promoters or enhancers).

It has been proposed that long non-coding regions combined with widespread occurrence of transposons may have facilitated the increase in diversity of the higher eukaryotic organisms (Alberts et al., 1994).

\section{The Artificial Genome}

Most of the GRN models mentioned in Section 3 use a network description as their base level of representation. In some cases this representation is translated into a sequence before the biologically inspired genetic algorithm operators can be applied. However, in this context, such genetic operators bear little if any real similarity to their biological counterparts. By starting with a se- 


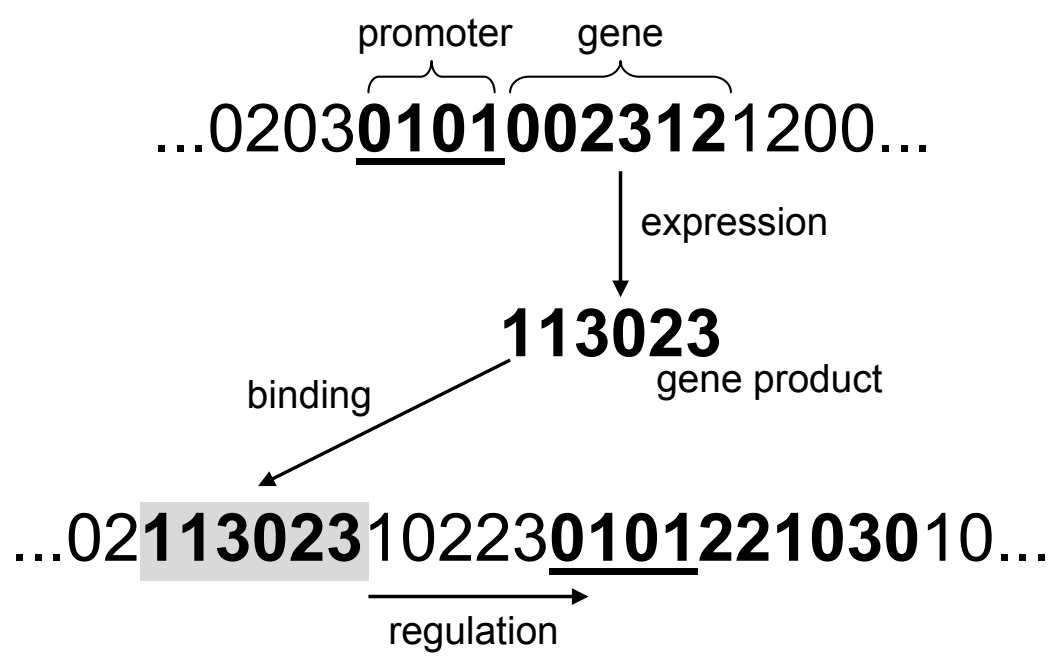

Fig. 2. The Artificial Genome model. Fixed length genes are defined by a 'promoter' sequence ('0101' in the example above). A gene is expressed by incrementing each element by 1 . The gene product then binds to matching sequences in the genome to regulate the next gene downstream.

quence as the base level of representation, and then extracting a network from this sequence, it becomes possible to incorporate a range of more meaningful mutation operators.

The Artificial Genome model developed by Torsten Reil is one such sequencebased model (Reil, 1999), which we have used to investigate the effects of mutation operators on network structure. In this model, a random string of bases is generated. This string is then searched for the promoter sequence '0101' and the following 6 digits are defined to be the gene sequence. The sequence between genes is defined to be the regulatory region for the following gene. A gene is expressed by incrementing each digit by 1 , modulo the number of bases (4). The regulatory region of each gene is then searched for matches to any of the gene products; each hit defines a regulatory link between the gene that produced the product and the gene in whose regulatory region the match was found (see Figure 2).

\section{Effects of mutation operators on network architecture}

In order to determine the influence of sequence-level alteration at the regulatory network level in the Artificial Genome, investigations were undertaken into the effects of the following five sequence-level mutation operators:

- Single-Point: A single random nucleotide changed to a different value

- Duplication: A random segment of the genome sequence is duplicated, with the copy being placed immediately after the original (i.e., a tandem dupli- 


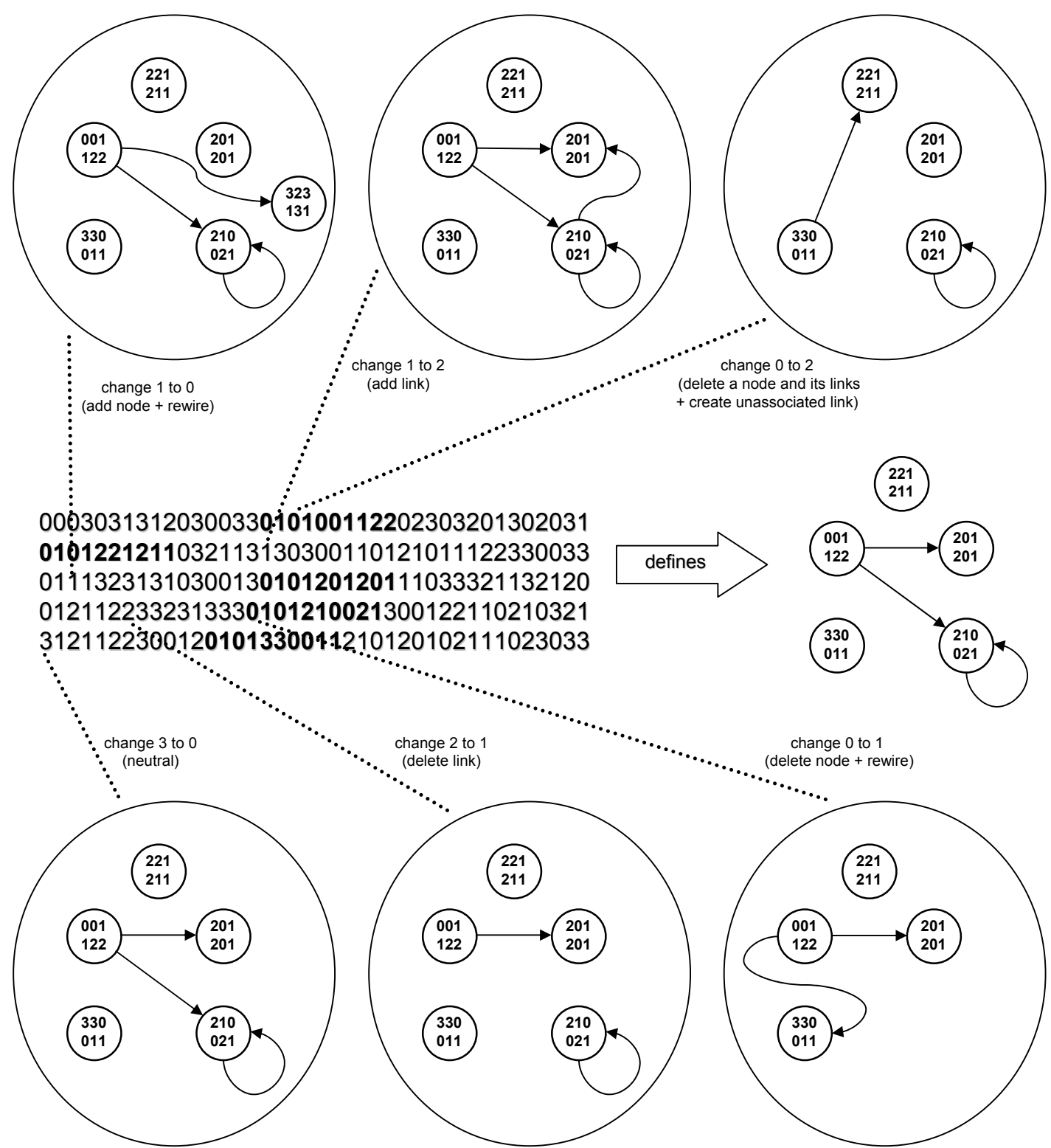

Fig. 3. Single-point mutations at the sequence level can cause a variety of changes at the network level. Although the vast majority of single-point mutations are neutral in a standard-size genome sequence, a small number of single value changes can have a significant impact on network composition.

cation). The size of the segment was 256 , so that, on average, a single gene is affected (the chance of a gene occurring depends on the probability of '0101' occurring).

- Transposition: A random 256 sized segment of the genome moved to another random location.

- Deletion: A random 256 sized segment of the genome removed.

- Inversion: The values of a random 256 sized segment of the genome rewritten in reverse order. 
Table 1

Total number of network effects across 100 sequence mutations

\begin{tabular}{|r|c|c|c|c|}
\hline \hline & Add Gene & Remove Gene & Add Link & Remove Link \\
\hline Single-Point & 3.64 & 3.68 & 22.88 & 22.52 \\
Duplication & 90.52 & 0.00 & 413.46 & 3.16 \\
Transposition & 9.08 & 8.90 & 410.24 & 408.16 \\
Deletion & 3.09 & 93.59 & 135.57 & 539.55 \\
Inversion & 93.70 & 93.09 & 573.92 & 568.79 \\
\hline
\end{tabular}

Effects of single-point mutations on network structure, averaged across 100 random sequences.

A series of simulations were used to determine the likelihood of network-level changes that could result from individual sequence-level mutations. Random sequences comprised of 10,000 "nucleotides" were generated. Regulatory networks were extracted by the process described in Section 4 (see Figure 2). In 100 random sequences, extracted networks had an average of 42.15 genes (standard deviation of 4.39) and average connectivity of 2.27 (standard deviation of 0.30$)$.

For each of the five mutation operators, the number of times a network alteration (i.e., gene addition, gene removal, link addition, link removal) occurred was recorded for 100 mutation events averaged over 100 random sequences (see Table 1). The number of times a network change occurred at least once for each given mutation operator was also recorded (see Figure 4).

Note that genes are defined in this model by their value. So that if a gene's value is changed, this is recorded as being a gene removed and a gene added. Similarly, links are defined by the genes they regulate. Thus, if a link from gene $\mathrm{A}$ to gene $\mathrm{B}$ is altered to become a link from gene $\mathrm{A}$ to gene $\mathrm{C}$, a link deletion and addition are recorded.

For single-point mutation, gene additions and deletions were rare (less than $4 \%$ of mutations), while links were added and removed just under $10 \%$ of the time. The vast majority of sequence changes were neutral (86\%). It is worth noting that many non-neutral single-point mutations affect multiple components at the network level, such as adding a node and rewiring, or deleting a node and rewiring (see Figure 3).

Transposition events added and removed genes just under $10 \%$ of the time, with links being influenced in $90 \%$ of the applications of this operator. Deletion events removed genes and links $61 \%$ and $94 \%$ of the time respectively. Interestingly, sequence deletions occasionally caused gene additions and often link additions. Note that most of these additions can be attributed to our 


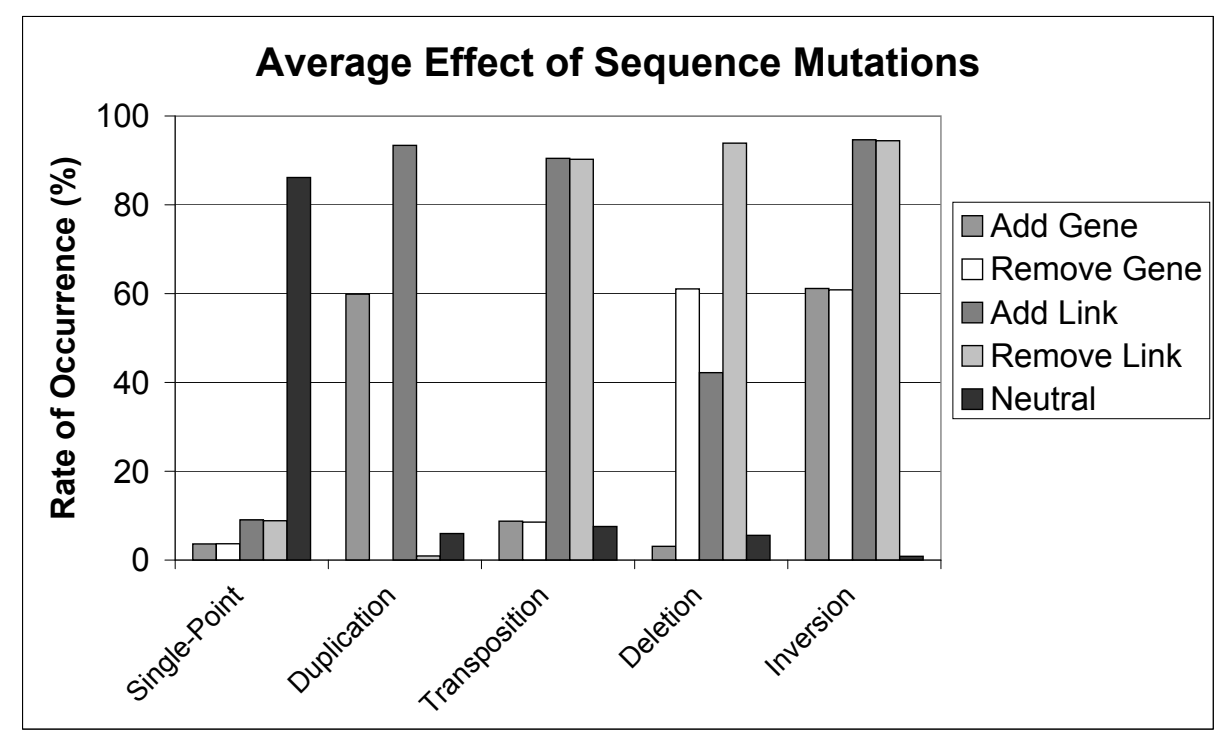

Fig. 4. Effects of various sequence-level changes at the network level. Note that this figure illustrates the number of times a given network operator occurs at least once. Note also that two events occurring in the same mutation event are recorded (e.g., a single change may both add a gene and delete a link).

method of defining genes and links (see above). Also for this reason, inversions are shown to have a significant impact on network structure.

Tandem duplication of a segment of the chromosome does not result in a 'pure' (in the network sense) gene duplication within the Artificial Genome model. Approximately $60 \%$ of the time, a sequence duplication adds a gene. Links are added nearly $94 \%$ of the time. Very occasionally, links are removed, and no genes were removed in 100 applications of this operator. Due to the way regulation is defined in the Artificial Genome model, both the original and the duplicate gene will have the same functionality and hence will regulate the same set of genes. Thus, in network terms, when a node is duplicated, its directed links to other nodes will be duplicated also. However, the duplication event is not limited to the sequence for the gene itself, and part of the regulatory regions both upstream and downstream of the gene are also likely to be duplicated. Such a sequence duplication produces an interesting transformation on the extracted network. Consider a hypothetical case in which half of the regulatory regions both upstream and downstream of a gene are duplicated. The new gene will share half of its regulatory region with its original gene (and hence share half of its directed links from other nodes), and the other half will be from the downstream gene, which could correspond to a completely unrelated sub-component of the genetic regulatory network. Effectively, the duplicated gene can be activated by two different contexts, that of the original and the downstream (unrelated) gene (see Figure 5). As a consequence, analysing possible changes due to gene duplication requires separating the influences of a duplication event on directed links into and out-of a node. 


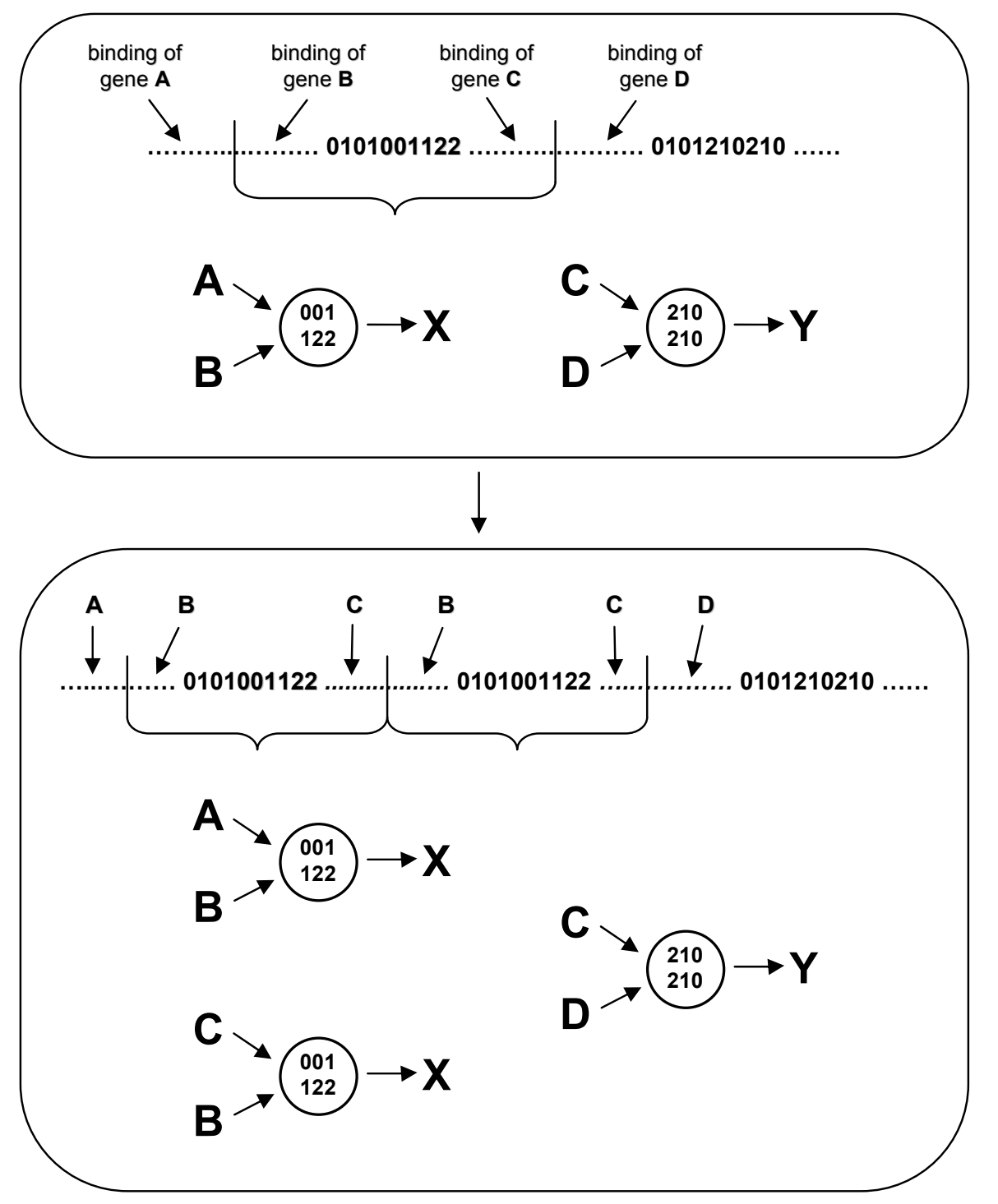

Fig. 5. Example network change after duplicating a portion of the nucleotide sequence. A typical duplication of a gene involves creating a copy of the gene and changing the set of genes that regulate it.

\section{Accumulated change}

Most important for the evolution of GRNs is the cumulative effects of mutations over time. By studying cumulative mutations, we can see how characteristics of the networks change independently of selection.

Statistically, accumulated single-point, inversion and transposition events over time are equivalent to creating new random networks. Accumulated deletions whittle away the genome until it is non-existent. More interesting is the accumulation of duplication events, since portions of the same sequence can occur multiple times. 


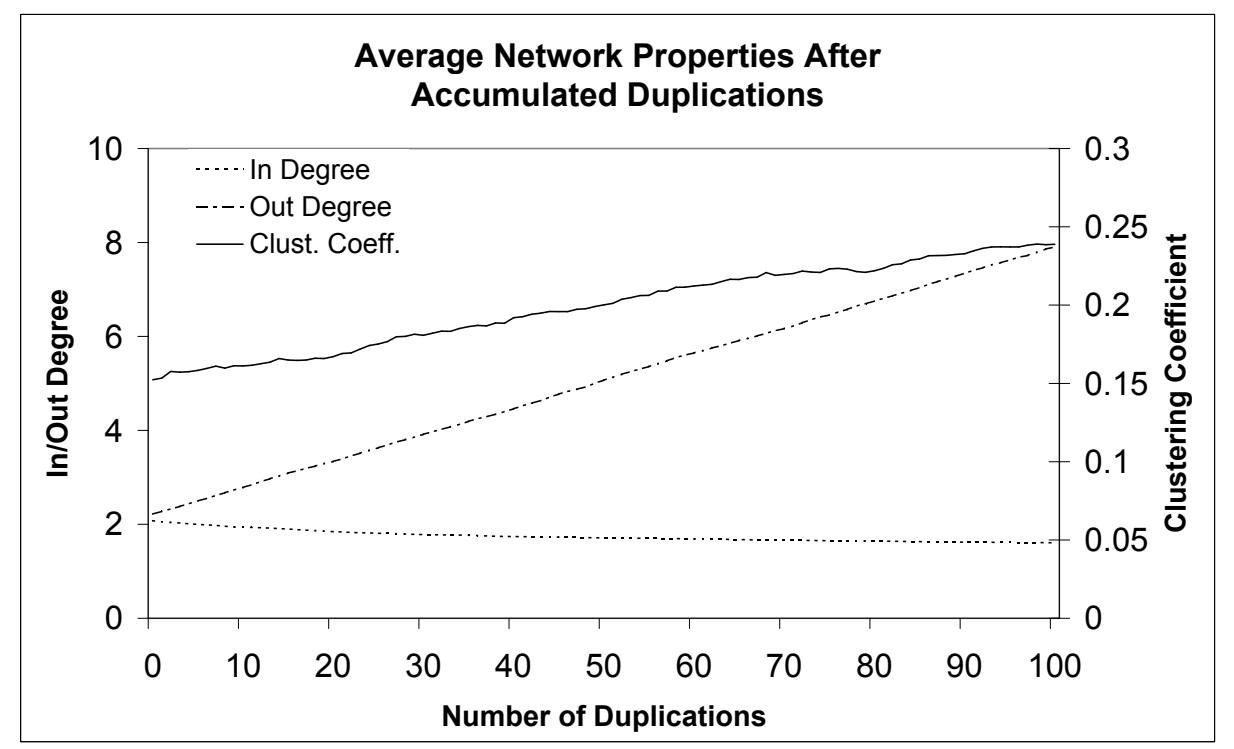

Fig. 6. Average network properties after accumulated tandem duplications. Note that as duplications are accumulated, the average out-degree linearly increases, while the in-degree decreases. Also note the increase in the average clustering coefficient. These results are averaged over 100 random sequences.

\subsection{Multiple duplications}

The effect of accumulated sequence duplications on network properties were investigated via simulation using the sequences described in Section 5. As above, segments of size 256 were randomly chosen for duplication. The results of these studies are summarised in Figure 6. As expected, the average number of genes and the average out-degree (the average number of genes regulated by each gene in the network) increased linearly as tandem duplications were accumulated. Interestingly, the average in-degree (i.e., average number of genes that regulate a gene in the network), decreases as duplications are accumulated. In addition, the average clustering coefficient increases. The clustering coefficient in this case is defined as the number of connections between neighbourhood nodes divided by the total number of possible connections that could exist between these neighbours. In our Boolean model, a neighbour of a gene is defined as any gene that is regulated by that gene.

\subsection{Multiple duplications with single-point mutations}

Interactions between duplication and single-point mutation events are likely to have been the most influential in generating the genetic regulatory networks of complex organisms. Presented here are the results of a study identical to the one described above in Section 6.1, with the addition of 10 random single-point mutations at each duplication event. (The locations of the single-point muta- 


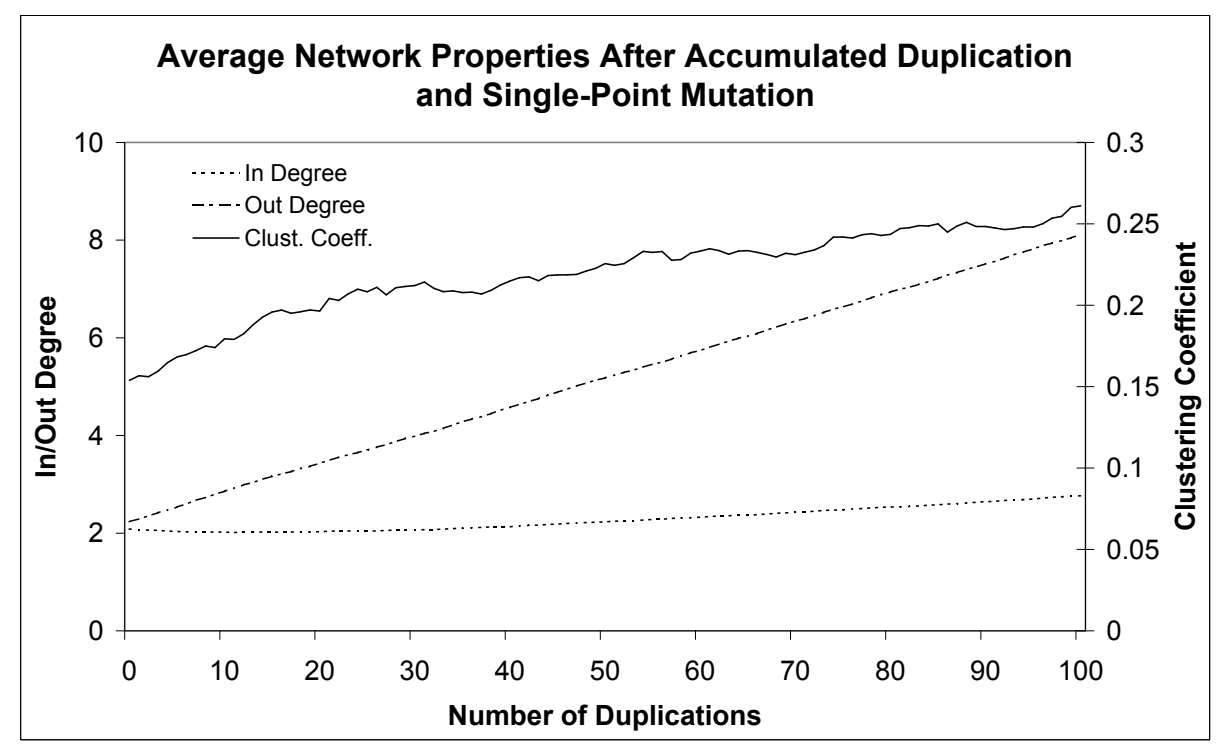

Fig. 7. Average network properties after accumulated tandem duplications, with 10 single-point mutations per duplication. Note the similarity with Figure 6, with the exception of the average in-degree (which increases). These results are averaged over 100 random sequences.

tions were randomly chosen from locations across the entire genome). Adding such single point mutations to sequence duplication events allows subsequent rewiring of the regulatory genes. In this case, average number of genes, indegree and out-degree are all basically equivalent to those found in Section 6.1. However, the average in-degree increased, rather than decreased, as shown in Figure 7.

\section{Discussion and conclusions}

Using the Artificial Genome model as a framework for describing genetic regulatory networks, we have explored the mapping between sequence level mutations and the effects they have on the resulting network structure. In particular, we have described how the frequency with which particular structural changes occur varies depending on the type of mutation applied.

We have shown that even single nucleotide changes can cause a wide variety of network-level changes in the Artificial Genome model. Transpositions, deletions and inversions caused interesting effects at the network level, it being rare that an isolated gene or link modification occurred. In particular, an interesting finding in this model is that gene duplication at the sequence level constrains the new gene to be regulated by a subset of existing regulators, which causes an increase in the clustering coefficient of the network over repeated duplications. This results in a very different network architec- 
ture to duplicating nodes and randomly rewiring at the network level. We have also demonstrated how the repeated application of gene duplication and single point mutation events can, over evolutionary time, result in significant changes to the network architecture.

When using artificial evolution to model systems such as genetic regulatory networks, it is critical that the mutation operators used capture the relevant features of their biological counterparts. In the domain of genetic regulatory networks, this means that the mutation operators chosen to evolve the networks should reflect the effects of biologically plausible mutations to the underlying genome sequences.

By using the Artificial Genome framework to extract networks from a sequence level representation, and implementing mutation operators at the sequence level, rather than the network level, we have achieved two things. Firstly, we have expanded the range of mutation operators that may be sensibly incorporated into evolutionary studies of genetic regulatory networks. Secondly, we have demonstrated that the Artificial Genome framework represents a systematic and principled approach to mapping a set of sequence level mutation operators onto their network level effects. The potential of this framework to model the effects of other types of genetic change, such as recombination, should be readily apparent.

\section{References}

Albert, R., Jeong, H., Barabási, A.-L., 2000. Error and attack tolerance of complex networks. Nature 406, 378-382.

Alberts, B., Bray, D., Lewis, J., Raff, M., Roberts, K., Watson, J. D., 1994. Molecular Biology of the Cell, 3rd Edition. Garland Publishing Inc., New York, NY.

Bornholdt, S., 2001. Modeling genetic networks and their evolution: A complex dynamical systems perspective. Biological Chemistry 382, 1289-1299.

Bornholdt, S., Rohlf, T., 2000. Topological evolution of dynamical networks: Global criticality from local dynamics. Physical Review Letters 84 (26), 6114-6117.

Bornholdt, S., Sneppen, K., 1998. Neutral mutations and punctuated equilibrium in evolving genetic networks. Physical Review Letters 81 (1), 236-239.

Bornholdt, S., Sneppen, K., 2000. Robustness as an evolutionary principle. Proceedings of the Royal Society of London, Series B 267, 2281-2286.

Dorogovtsev, S. N., Mendes, J. F. F., 2002. Evolution of networks. Advances in Physics 51 (4), 1079-1187.

Frank, S. A., 1999. Population and quantitative genetics of regulatory networks. Journal of Theoretical Biology 197, 281-294. 
Jeong, H., Tombor, B., Albert, R., Oltvai, Z. N., Barabási, A.-L., 2000. The large-scale organization of metabolic networks. Nature 407, 651-654.

Kauffman, S. A., 1971. Gene regulation networks: A theory for their global structure and behaviours. Current Topics in Developmental Biology 6, 145182.

Kauffman, S. A., 1990. Requirements for evolvability in complex systems: Orderly dynamics and frozen components. Physica D 42, 135-152.

Kauffman, S. A., 1993. The Origins of Order: Self-Organization and Selection in Evolution. Oxford University Press, Oxford, UK.

Kauffman, S. A., Smith, R. G., 1986. Adaptive automata based on Darwinian selection. Physica D 22, 68-82.

Lynch, M., 2002. Gene duplication and evolution. Science 297, 945-947.

Mitchell, M., 1996. An Introduction to Genetic Algorithms. The MIT Press/Bradford Books, Cambridge, MA.

Ohno, S., 1970. Evolution by Gene Duplication. Springer-Verlag, Berlin.

Pastor-Satorras, R., Smith, E., Solé, R. V., 2003. Evolving protein interaction networks through gene duplication. Journal of Theoretical Biology 222, 199210.

Reil, T., 1999. Dynamics of gene expression in an artificial genome - implications for biological and artificial ontogeny. In: Floreano, D., Nicoud, J.-D., Mondada, F. (Eds.), Advances in Artificial Life: 5th European Conference (ECAL 99). Vol. 1674 of LNAI. Springer-Verlag, Berlin, pp. 457-466.

Solé, R. V., Goodwin, B., 2000. Signs of Life: How Complexity Pervades Biology. Basic Books, New York, NY.

Solé, R. V., Pastor-Satorras, R., Smith, E., Kepler, T. B., 2002. A model of large-scale proteome evolution. Advances in Complex Systems 5 (1), 43-54.

Stern, M. D., 1999. Emergence of homeostasis and "noise imprinting" in an evolution model. Proceedings of the National Academy of Science, USA 96, 10746-10751.

Strogatz, S., 2001. Exploring complex networks. Nature 410, 268-276.

Vázquez, A., Flammini, A., Maritan, A., Vespignani, A., 2001. Modelling of protein interaction networks, cond-mat/0108043.

Wagner, A., 1994. Evolution of gene networks by gene duplication: A mathematical model and its implications on genome organization. Proceedings of the National Academy of Science, USA 91, 4387-4391.

Wagner, A., Fell, D. A., 2001. The small world inside large metabolic networks. Proceedings of the Royal Society of London, Series B 268, 1803-1810.

Watts, D. J., 1999. Small Worlds: The Dynamics of Networks between Order and Randomness. Princeton University Press, Princeton, NJ.

Wolf, Y. I., Karev, G., Koonin, E. V., 2002. Scale-free networks in biology: New insights into the fundamentals of evolution? BioEssays 24, 105-109.

Wuensche, A., 1998. Genomic regulation modeled as a network with basins of attraction. In: Altman, R. B., Dunker, A. K., Hunter, L., Klein, T. E. (Eds.), Pacific Symposium on Biocomputing '98. World Scientific, Singapore, pp. 89-102. 